\title{
BadgerFilm: An Open Source Thin Film Analysis Program
}

Aurélien Moy ${ }^{1}$ and John Fournelle ${ }^{2}$

${ }^{1}$ Department of Geoscience, University of Wisconsin-Madison, Madison, Wisconsin, United States, ${ }^{2}$ University of Wisconsin, Madison, Madison, Wisconsin, United States

The characterization of layered specimens by electron probe microanalysis (EPMA) has been attempted since the early developments of the field in the 60's [1]. It is only since the development of the second generation of matrix corrections, with accurate $\varphi(\rho z)$ ionization depth distributions, in the 90's, that the method significantly improved. The most recent $\varphi(\rho z)$ models - PROZA96 [2], PAP and XPP [3], and XPHI [4] - realistically describe the ionization depth distributions in bulk homogeneous materials. However, the models cannot be directly used to quantify thin film samples as the physics behind greatly differs between stratified and bulk samples. These models have been adapted by their authors to describe the $\varphi(\rho z)$ distributions in stratified samples by different weighting procedures, allowing good quantification results of the thickness and composition of the different layers. However, thin film analysis programs are not commonly available in most laboratories, mainly because the characterization of stratified samples is not a routine task and also because these programs, with exception of GMRFilm [5], are not free. GMRFilm is a research grade, shareware DOS program for thin film analysis created by R. Waldo at General Motors Research Labs in the beginning of the 90's. However, despite its great performances, this program has some limitations, such as not being able to bulk process data acquired at several accelerating voltages, and because of its "advanced age", lacks flexibility. In addition, modern operating systems tend to not be natively compatible with old DOS programs, making its use more complicated.

This led us to the creation of a modern thin film analysis program, flexible and open source, named BadgerFilm. The program, developed in Visual Basic .NET, is composed of a simple graphical interface, implements the most recent $\varphi(\rho z)$ models, as well as a non-linear fitting algorithm to determine compositions and thicknesses of stratified specimens. The program calculates theoretical k-ratios (the ratio of the X-ray intensity emitted by the unknown by that of a standard) and matches them to the experimental k-ratios by modifying the compositions and thicknesses of the studied system.

Testing the performance and predictions of the thin film algorithms is a difficult matter as thin film standards are very scarce. Monte Carlo simulations are a good alternative to assess the accuracy of the predictions. By using the same atomic parameters (mass absorption coefficients, ionization cross sections, transition probabilities, ...) as the ones used in the Monte Carlo code PENELOPE/PENEPMA [6] in our implementation of the PAP $\varphi(\rho z)$ model, we found that calculated characteristic X-ray intensities, in absolute value, can directly be compared to the Monte Carlo predictions. Using our implementation of the PAP model and using the Monte Carlo code PENEPMA, X-ray intensities emitted from bulk materials (pure or compounds) and thin film on substrate specimens (with various compositions and thicknesses) were calculated for electron beam energies ranging from 5 to $30 \mathrm{keV}$. The predicted absolute primary Xray intensities were very similar for bulk samples and as well as for stratified specimens (Figure 1).

In many cases, secondary fluorescence emitted either by the primary characteristic X-rays or by the bremsstrahlung cannot be neglected when calculating the composition and thickness of stratified specimens. The fluorescence of the film by the characteristic X-rays coming from other layers or from the substrate can increase the X-ray intensity emitted from the film by several tenth of a percent. As an 
example, the Fe Ka X-ray intensity emitted by a 100-nm thick film of FeSi2 on top of a $\mathrm{Cu}$ substrate is increased by $8 \%$ at $25 \mathrm{kV}$ due to fluorescence by the $\mathrm{Cu} \mathrm{K} \alpha-\mathrm{K} \beta \mathrm{X}$-ray lines. Secondary fluorescence produced by the bremsstrahlung is also able to increase the emitted X-ray intensity by a few percent particularly when the energy of the beam is much greater than the studied characteristic X-ray energy. In the previously mentioned example, the contribution of the secondary fluorescence created by the bremsstrahlung to the total Fe K $\alpha$ X-ray intensity is about $2 \%$ at $25 \mathrm{kV}$. The total secondary fluorescence accounts for $10 \%$ of the total $\mathrm{Fe} \mathrm{K} \alpha \mathrm{X}$-ray intensity and thus can lead to similar errors in the determination of the film thickness and composition.

BadgerFilm takes into account all the characteristic X-rays that have an energy higher than the ionization threshold of the studied X-ray line in the calculation of the secondary fluorescence due to characteristic X-rays. $\varphi(\rho z)$ distributions for all these characteristic X-rays are calculated and integrated to determine their contribution to the secondary fluorescence. As seen on Figure 2, the secondary fluorescence produced by characteristic X-rays and calculated by BadgerFilm is in good agreement with the Monte Carlo simulations.

Bremsstrahlung secondary fluorescence is more difficult to calculate because these X-rays are not emitted at one single characteristic energy, but they form a continuum ranging from $0 \mathrm{eV}$ up to the energy of the electron beam. For each X-ray energy, the depth distribution is not well described by analytical theories. These distributions can be calculated by Monte Carlo simulations [7], but it is generally too time consuming. A method to approximate these distributions consists of calculating the X-ray depth distribution emitted by a fictitious element that has a characteristic X-ray energy corresponding to the considered bremsstrahlung X-ray energy. This rough approximation, which is used in the GMRFilm program, tends to overestimate the calculated secondary fluorescence. However, by applying two empirical correction factors, based on the element and sample studied, the calculations are in good agreement with the results obtained by Monte Carlo simulations (Figure 2) [8].

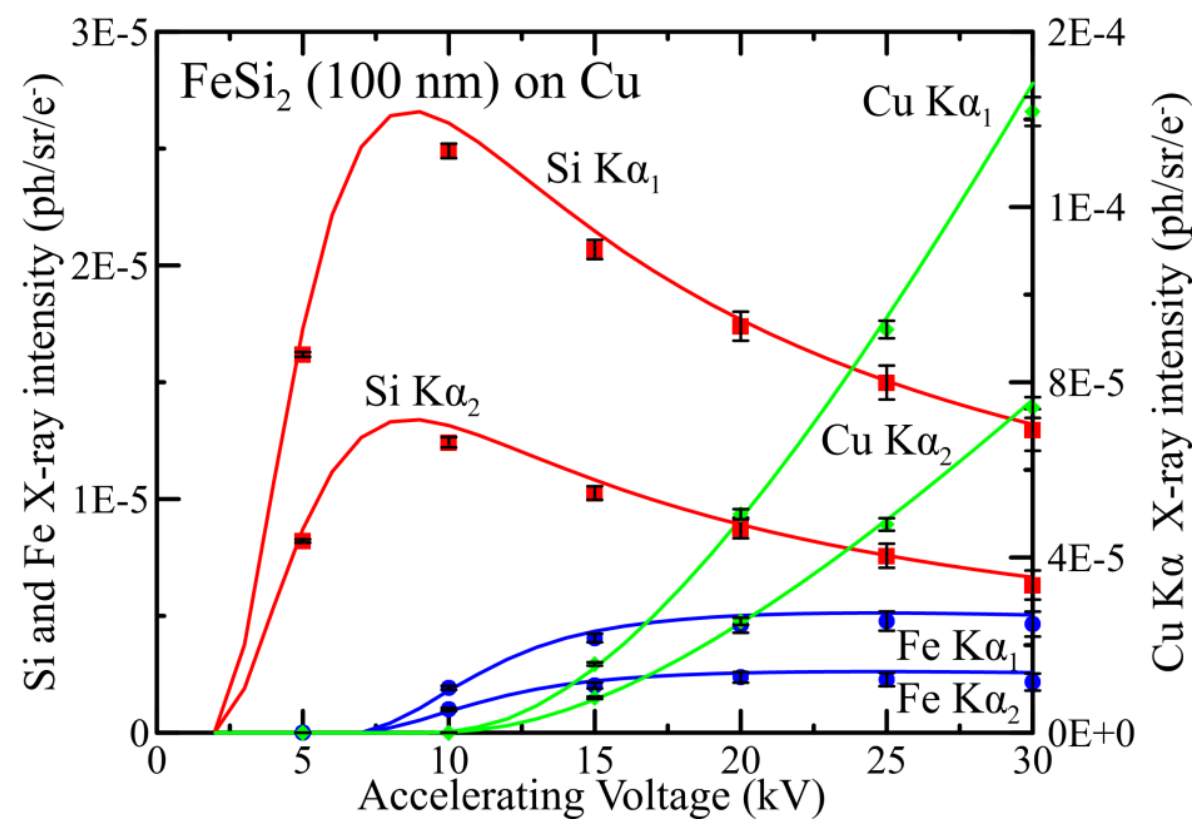

Figure 1. Figure 1: Primary characteristic X-ray intensities calculated with the PAP model (lines) and PENEPMA (symbols), using the same atomic parameters. 


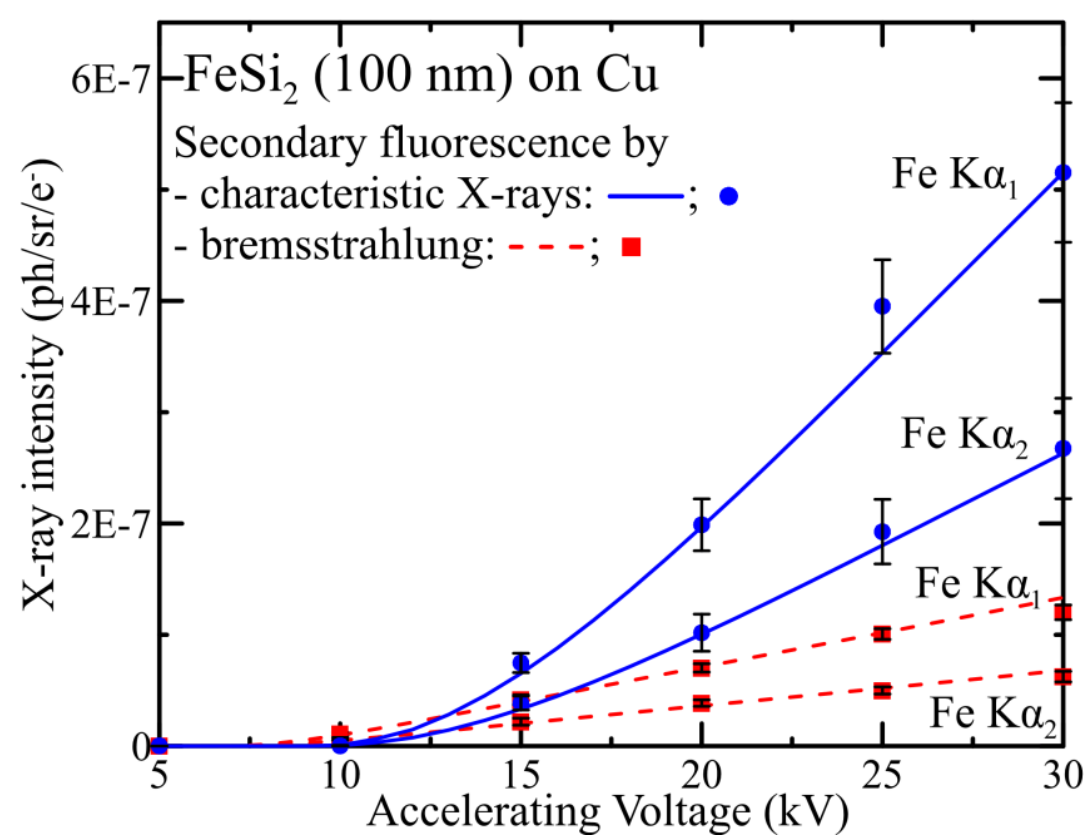

Figure 2. Figure 2: Secondary fluorescence calculated with our modified implementation of the PAP model (lines) and PENEPMA (symbols), using the same atomic parameters.

\section{References}

[1] W. E. Sweeney Jr., R. E Seebold and L. S. Birks, "Electron Probe Measurements of Evaporated Metal Films", Journal of Applied Physics 31, 1061 (1960), p. 1061-1064.

[2] G. F. Bastin, J. M. Dijkstra and H. J. M. Heijligers, X-ray Spectrometry 27, 1 (1998), p. 3-10.

[3] J. L. Pouchou and F. Pichoir, "Quantitative analysis of homogeneous or stratified microvolumes applying the model PAP”, in Electron Probe Quantitation. Plenum Press (1991), p. 31.

[4] C. Merlet, Mikrochimica Acta 114/115 (1994), p. 363.

[5] R. A. Waldo, "An Iteration Procedure to Calculate Film Compositions and Thicknesses in ElectronProbe Microanalysis”, in Microbeam Analysis. San Francisco Press (1988), p. 310.

[6] X. Llovet and F. Salvat, IOP Conference Series: Materials Science and Engineering 109 (2016).

[7] Y. Yuan et al., Microscopy and Microanalysis 25, 1 (2019), p. 92-104.

[8] Support for this research came from the National Science Foundation: EAR-1337156 (JHF), EAR1554269 (JHF) and EAR-1849386 (JHF). 